Nervenarzt 2020 $91: 161-169$

https://doi.org/10.1007/s00115-019-00865-0

Online publiziert: 3. Februar 2020

(c) Der/die Autor(en) 2020
CME

Zertifizierte Fortbildung

\section{Importierte Infektionen des Zentralnervensystems}

Migrations- und Reise-Neurologie

\section{Erich Schmutzhard · Bettina Pfausler}

Universitätsklinik für Neurologie, Medizinische Universität Innsbruck, Innsbruck, Österreich
Online teilnehmen unter:

www.springermedizin.de/cme

Für diese Fortbildungseinheit werden 3 Punkte vergeben.

\section{Kontakt}

Springer Medizin Kundenservice Tel. 08007780777

(kostenfrei in Deutschland)

E-Mail:

kundenservice@springermedizin.de

\section{Informationen}

zur Teilnahme und Zertifizierung finden Sie im CME-Fragebogen am Ende des Beitrags.

\section{Zusammenfassung}

Die Zahl der MigrantInnen und Flüchtlinge erreichte in Mitteleuropa in den Jahren 2015 und 2016 ein „Allzeithoch“. Sie ist mittlerweile wieder auf Durchschnittszahlen der letzten 30 Jahre zurückgegangen. Demgegenüber zeigen die Zahlen der internationalen und interkontinentalen Reisenden über nun bereits Jahrzehnte eine kontinuierliche Zunahme. Weltweit werden von internationalen Organisationen mehr als 1,35 Mrd. „incoming travellers" berichtet. Die genaue Kenntnis der Epidemiologie akuter oder chronischer Infektionen des Zentralnervensystems (ZNS) erlaubt frühzeitig die richtigen, möglicherweise lebensrettenden diagnostischen und therapeutischen Schritte. Akute ZNS-Infektionen, wie zerebrale Malaria oder arbovirale Enzephalitis, werden überwiegend bei Reiserückkehrern gesehen, während chronische Infektionen und Infestationen des ZNS, wie tuberkulöse Meningits oder Neurozystizerkose, typischerweise bei MigrantInnen, Asylwerbern und Flüchtlingen auftreten. Neben ZNSInfektionen müssen auch genetisch bedingte, umweltassoziierte, nutritionsbedingte oder metabolische Erkrankungen in der Differenzialdiagnose berücksichtigt werden.

\section{Schlüsselwörter}

Migration · Arbovirale Enzephalitis · Tuberkulose · Neurozystizerkose · Malaria · Akute und chronische ZNS-Infektionen

\section{Lernziele}

\section{Nach Lektüre dieses Beitrags ...}

- können Sie wichtige importierte neurologische Erkrankungen benennen,

- ziehen Sie korrekte Schlüsse aus der klinischen Präsentation importierter Erkrankungen des Zentralnervensystem (ZNS), wie z. B. Neurozystizerkose,

- erkennen Sie lebensbedrohliche importierte ZNS-Infektionen wie zerebrale Malaria,

- verwenden Sie antiplasmodiale und anthelminthische Therapeutika sicher. 


\section{Hintergrund}

Im Jahr 2018 unternahmen 1.350.000.000 Menschen eine Reise über eine Grenze hinweg (,international tourist arrivals"), die Zahlen steigen um $6-7 \%$ pro Jahr $[1,2]$.

Weltweit ist die Zahl der MigrantInnen laut UNO in den letzten 10 Jahren um ein Viertel gestiegen, von 221 Mio. auf 272 Mio., es sind somit heute 51 Mio. mehr als 2010 (Mitteilung auf der Homepage des für Bevölkerungsfragen zuständigen UNO-Büros für wirtschaftliche und soziale Angelegenheiten [3] am 17.09.2019). Die meisten dieser MigrantInnen leben in Europa (81 Mio.) und in Nordamerika (59 Mio.), je 49 Mio. in Nordafrika und Westasien. Die Hälfte dieser 272 Mio. Menschen lebt dem Bericht zufolge in gerade einmal 10 Ländern. Die USA liegen mit 51 Mio. an der Spitze. Mit großem Abstand folgen Deutschland und Saudi-Arabien mit jeweils 13 Mio., Russland mit 12 Mio., Großbritannien mit 10 Mio. und die Vereinigten Arabischen Emirate mit 9 Mio. In Frankreich, Kanada und Australien leben jeweils 8 Mio. und in Italien 6 Mio. Einwanderer.

Bei den Herkunftsländern der Migranten liegt Indien mit 18 Mio. Auswanderern an der Spitze, gefolgt von Mexiko mit 12 Mio., China mit 11 Mio., Russland mit 10 Mio. und Syrien mit 8 Mio. MigrantInnen.

Das Bundesamt für Migration und Flüchtlinge publizierte im März 2019 folgende Zahlen bez. Flüchtlinge, Asylsuchende/-bewerberlnnen in Deutschland: Im 65-Jahres-Zeitraum 1953 bis 2018 wurden ca. 5,8 Mio. Asylanträge registriert, davon 4,9 Mio. (84\%) im Zeitraum 1990 bis 2018. Die Dynamik der Zahlen der Asylanträge spiegelt die regionale, aber auch weltweite politische und wirtschaftliche Situation wider:

- 1992:438.191,

- 1995: 166.951,

- 2005:42.908,

- 2015:476.849,

- 2016:745.541,

- 2017:222.693,

- 2018: 185.853 .

Reisende in bzw. MigrantInnen/Flüchtlinge aus tropischen und/ oder subtropischen Regionen können sich - im Krankheitsfall - mit einer Vielzahl lokaler und regionaler Besonderheiten präsentieren; diese zu erkennen und richtig einzuordnen setzt detailliertes klinisches und epidemiologisches Wissen voraus. Neben "exotischen" Infektionskrankheiten muss bei neurologischen Symptomen auch an Malnutrition, Wasser- und Elektrolytmangel gedacht werden und besonders während der Migration oder in Flüchtlingslagern Nordafrikas oder des Vorderen Orients an physische und psychische Gewalt $[4,5,6,7,8,9,10,11,12,13$, 14]. Eine detaillierte Anamnese bez. der Exposition gegenüber Vektoren, Arthropoden (Insekten oder Zecken), Ratten, Mäuse etc., ist meist sehr schwierig, aber in Notfallsituationen unerlässlich $[15,16,17,18$, 19]. Die behandelnden NeurologInnen sind gefordert, wesentliche epidemiologische Grundkenntnisse über die geographische Verbreitung humanpathogener Erreger, Übertragungswege (Arthropoden, Tierbiss oder direkt von Mensch zu Mensch), Anamnese in Bezug auf Infektionserreger, aber auch Impfstatus oder Resis-
Imported infections of the central nervous system. Migration and travel neurology

The numbers of migrants, refugees and asylum seekers reached an unprecedented high in Europe in 2015 and 2016 but in 2019 they are back to the average numbers of the last 30 years. In contrast, frequencies of international and intercontinental travelers have continuously increased over the past decades and will continue to do so in the coming years. In 2018 more than 1.35 billion incoming travelers were reported worldwide by international organizations. Detailed knowledge of the epidemiology, transmission types, risk behavior and clinical presentation of acute and chronic central nervous system (CNS) infections enables timely diagnosis and initiation of potentially lifesaving emergency treatment. Acute infections of the CNS, e.g. cerebral Plasmodium falciparum malaria or arboviral encephalitis, are seen most frequently and almost exclusively in travelers returning from tropical countries, whereas chronic CNS infections, e.g. tuberculous meningitis or neurocysticercosis, are typically seen in migrants and refugees. Beside CNS infections genetic diseases, environment-associated, nutrition-related, metabolic or cerebrovascular diseases also need to be considered when discussing differential diagnostic possibilities.

Keywords

Migration - Arboviral encephalitis - Tuberculosis - Neurocysticercosis . Malaria $\cdot$ Acute and chronic CNS-infections

tenzsituationen (z.B. Mycobacterium tuberculosis) zu kennen und sich raschest möglich zu informieren [18, 19, 20, 21, 22, 23, 24]. Die häufig bestehende Sprachbarriere ist oft zusätzlich kompliziert durch kulturelle Kommunikationsbarrieren [25].

$>$ Merke

Die Zahl der Fernreisenden ist ein Vielfaches größer als die Zahl der Flüchtlinge.

Merke

Auch an „Nichtinfektions"-Krankheiten sollte gedacht werden.

\section{ZNS-Infektionen, Fernreise und Migration}

Brinckmann et al. berichten anhand einer konsekutiven Kohorte neurologischer Notfälle in der Notaufnahme der Charité Berlin mehrere Besonderheiten, wenn sie Menschen ohne Migrationshintergrund (NonmigrantInnen, N), MigrantInnen (M) und Flüchtlinge $(F)$ vergleichen [4]. Sie fanden folgende statistisch signifikante Unterschiede:

- M hatten seltener eine medizinische Vorgeschichte als N.

- F bekamen seltener eine Magnetresonanztomographie (MRT) als $\mathrm{N}$.

- Die Zeit bis zur Diagnose war bei F signifikant länger als bei M oder N.

- Eine Notfallmedikation wurde bei $\mathrm{F}$ signifikant seltener als bei $\mathrm{N}$ verabreicht.

- Eine stationäre Aufnahme wurde bei F signifikant seltener als bei $N$ für notwendig erachtet.

- Folgende Notfallbeschwerden/-symptome wurden berichtet:

- definitive Kopfschmerzen bei M signifikant häufiger als bei $\mathrm{N}$,

- nicht näher eingrenzbarer Kopfschmerz bei $\mathrm{F}$ signifikant häufiger als bei $\mathrm{N}$, 
Eine 23-jährige Medizinstudentin kehrt von einem 3-monatigen Thailandaufenthalt zurück. Sie hatte sich im Vorfeld der empfohlenen Reise Impfungen (Japanische Enzephalitis, Tollwut, Typhus - bei sonst vollständigem Impfstatus) unterzogen. Nach einem 4-wöchigen Aufenthalt in Bangkok (Famulatur) reiste sie mit lokalen Verkehrsmitteln durch das ganze Land. Außer einer 2-maligen, jeweils 3 Tage dauernden und selbst-limitierten Episode einer nichtblutigen Diarrhö war sie nie wirklich krank. Etwa 2 Wochen nach ihrer Rückkehr entwickelte sie eine Pollakisurie und wenige Tage später auch eine Sensibilitätsstörung in Nabelhöhe. Der Hausarzt überwies sie an einen Neurologen mit der Verdachtsdiagnose Multiple Sklerose.

Im neurologischen Status fand sich eine deutliche Reflexsteigerung an den unteren Extremitäten (Patellarsehnenreflex [PSR] rechts, Achillessehnenreflex [ASR] beidseits), allerdings war der PSR links auffällig abgeschwächt und die Patientin gab geringe, radikuläre Schmerzen, am ehesten einem radikulären Syndrom L (Lendenwirbel) 3/4 entsprechend an sowie einen positiven Babinski-Reflex beidseits; ab Th (Thoraxwirbel) 10 fand sich eine diskrete Hypästhesie. Im Bereich der oberen Extremitäten und der Hirnnerven sowie der höheren und höchsten kortikalen Funktionen fanden sich keinerlei Auffälligkeiten, allerdings zeigte die Patientin, bei milden Kopfschmerzen, einen geringgradigen Meningismus. Um die bei dieser Konstellation eher unwahrscheinliche Zuweisungsdiagnose Multiple Sklerose „auszuschließen“ wurde eine spinale und zerebrale MRT durchgeführt. In Höhe des unteren Thorakalmarkes zeigte das T2-gewichtete MRT eine Hyperintensität, die als transverse Myelitis interpretiert wurde. Eine Wurzel- oder Myelonkompression war auszuschließen. In der nun durchgeführten Lumbalpunktion zeigte sich eine eosinophile Pleozytose (210 Zellen/ul, 33 \% Eosinophile). Im Nativpräparat konnten einzelne "Larvae migrantes" visualisiert werden, die parasitologisch als Gnathostoma spinigerum identifiziert wurden. Eine ZNS-Gnathostomiasis ist die häufigste Ursache einer eosinophilen Radikulomyelitis in Südostasien, vor allem Thailand. Die infektiösen Larven werden durch die Ingestion unzureichend gekochter Muscheln, Schalentiere oder Fische aufgenommen. Die Patientin wurde mit Albendazol und Steroiden erfolgreich therapiert.
- Verdacht auf epileptischen Anfall bei F signifikant häufiger als bei $\mathrm{M}$ oder $\mathrm{N}$,

- möglicher oder definitiver epileptischer Anfall bei F signifikant häufiger als bei M und N.

Alle anderen Diagnosen und Beschwerden, insbesondere auch lebensbedrohliche Diagnosen, wie Schlaganfall oder symptomatische/sekundäre Kopfschmerzen, zeigten keinen Unterschied [4].

Diese Auflistung berücksichtigt nicht die überaus große Population der Tropenreisenden, Touristen, Geschäftsreisenden etc., die sich naturgemäß mit einem völlig unterschiedlichen Spektrum an neurologischen Notfällen präsentieren. Neben Einzelfällen von langstreckenflug- und/oder exsikkationsassoziierten Sinusthrombosen [26] oder tiefen Beinvenenthrombosen mit paradoxen Embolien sind es vor allem akute oder perakute Infektionskrankheiten des Zentralnervensystems (ZNS), wie zerebrale Malaria, virale Meningoenzephalitis, Helmintheninfestationen des ZNS, aber auch akute bakterielle Meningitis oder Hirnabszess [12, 18, 19, 20, 21, 22, 23, 24, 27, 28, 29, 30].

Demgegenüber präsentieren sich MigrantInnen, Asylwerberlnnen oder Flüchtlinge häufig spät und mit chronifizierten Beschwerden $[5,9,15,16,17,18]$. Allerdings kann natürlich jede/r Migrantln akut an einer lokal erworbenen Infektion des ZNS (z. B. Frühsommer-Meningoenzephalitis oder Neuroborreliose) erkranken oder unabhängig vom Migrantenstatus an einer sonstigen neurologischen Erkrankung leiden. MigrantInnen können auch (gesunde) "Carrier" eines Erregers sein, selbst erkranken oder andere Menschen „infizieren“; auch Zugvögel und andere Tiere sind als Carrier von Infektionserregern identifiziert worden (z. B. West-Nil-Virus), auch Vektoren migrieren (Arthropoden - Stechmücken oder Zecken), mit steigender Zahl von MigrantInnen gibt es auch eine höhere Wahrscheinlichkeit, genetisch bedingte „europaatypische" Erkrankungen zu sehen, z. B. Schlaganfall bei Sichelzellanämie, und letztlich sind auch soziale, kulturelle, religiöse, wirtschaftlich prekäre Faktoren bei Anamneseerhebung sowie diagnostischen oder therapeutischen Maßnahmen zu berücksichtigen [10, 15, 29, 31]. Gerade bei chronischen Infektionen des ZNS, z.B. ZNS-Tuberkulose, ist die Resistenzsituation der Erreger im Herkunftsland genauestens zu eruieren [16, 17, 18, 19, 27].
> Merke

Die genaue Anamnese ist trotz vielfältiger Barrieren in der Kommunikation unverzichtbar. Die Häufigkeit bestimmter Erkrankungen in den Herkunfts- bzw. Zielländern erlaubt schon frühzeitige, wichtige differenzialdiagnostische Überlegungen: Weltweit, vor allem in Südost- und Südasien sind Tollwutviren die häufigsten Auslöser einer tödlichen viraIen Enzephalitis. Dabei darf „Doktor Google“ befragt werden: d. h. nachschauen, nachlesen!

\section{Ursachenorientierte Gliederung importierter neurologischer Erkrankungen}

Die wesentlichen Ursachen neurologischer Erkrankungen bei dieser rasch größer werdenden Gruppe von PatientInnen sind [25, 29]:

- Infektionen und Infestationen,

- Malnutrition,

- klima- und temperaturbedingte Erkrankungen,

- giftige Tiere und tierische Gifte,

- umweltbedingte Erkrankungen,

- genetisch bedingte Erkrankungen,

- reduzierter medizinischer Standard.

Viele Infektionserreger (d.h. eine enorme Zahl von Viren, Bakterien, aber auch Pilzen, Protozoen, Helminthen oder Arthropoden) können oft nur mit ausreichendem Wissen über die Herkunft des/r Patienten/In - auch kurze Reisezwischenaufenthalte sind zu berücksichtigen -, die Transitroute, das epidemiologische "Setting", inklusive Inkubationszeiten, epidemischem Auftreten auch nichtneurologischer Manifestationen, die gesamte Akut- und Langzeitanamnese und die klinische Präsentation eruiert und entsprechend präzise diagnostiziert und gezielt therapiert werden. Solche Schwierigkeiten werden in der Kasuistik dargestellt.

Neurologische Infektionskrankheiten präsentieren sich häufig als Notfall mit einer sich rasch verschlechternden Prognose bei Diagnose- und Therapieverzögerung. Ausreichende Kenntnisse in der Epidemiologie erleichtern gezieltes Fragenstellen nach einer krankheitsweisenden Exposition (Malaria, Tollwut, Arboviren etc.), aber auch Inkubationszeiten und ein eventuell charakteristischer (monophasisch vs. biphasisch) Verlauf geben Hinweise. 


\section{Infobox 1}

Ausgewählte, hauptsächlich durch Infektionen ausgelöste neurologische Syndrome in der Tropenmedizin. (Mod. nach [29])

- (Akute) Bewusstseinsstörung mit Fieber

- Epilepsie/epileptische Anfälle

- Akuter Kopfschmerz

- Akute(r) Nackensteifigkeit/Meningismus

- Syndrom der chronischen Meningitis/Enzephalitis

- Hirnnervenneuritis

- Akute Querschnittssymptomatik

- Chronisch progrediente Querschnittssymptomatik

- Extrapyramidal motorische Symptomatik

- Polyneuritis, -neuropathie; Radikulitis

- Myositis

Als Orientierungshilfe sind - syndromorientiert - die wesentlichen Infektionskrankheiten in Infobox 1 [29] aufgelistet; sie werden im Folgenden schlagwortartig besprochen.

\section{Akute Bewusstseinsstörung/Koma mit Fieber}

Zerebrale Malaria, eitrige Meningitis, virale Meningoenzephalitis und hitzeassoziierte/-bedingte Erkrankungen (Hitzschlag/ Hyperprexie): Folgendes „notfallneurologische“ Vorgehen wird empfohlen.

Erster Schritt: Fundoskopie mit folgenden Fragen:

- Liegt eine Malaria-Retinopathie [33] vor? $\rightarrow$ eine zerebrale Malaria abklären.

- Liegt ein Papillenödem vor? $\rightarrow$ bei erhöhtem Hirndruck diffuses Hirnödem, Enzephalitis, bakterielle Meningitis, Pyozephalus, Hydrozephalus, Zerebritis, Abszess abklären.

- Liegen retinale Tuberkulome vor? $\rightarrow$ eine ZNS-Tuberkulose abklären.

Zweiter Schritt: Zerebrale Bildgebung (kraniale Computertomographie [cCT] und/oder kraniale MRT) mit folgenden Fragen durchführen:

- Liegt ein diffuses Hirnödem vor? $\rightarrow$ an Enzephalitis, Meningitis, zerebrale Malaria denken.

- MRT-verdächtig auf posteriores reversibles Enzephalopathiesyndrom (PRES)? $\rightarrow$ an zerebrale Malaria denken.

- Liegt eine meningeale Anreicherung nach i.v. Kontrastmittel vor? $\rightarrow$ an bakterielle Meningitis denken.

- Liegt ein akuter Hydrozephalus vor? $\rightarrow$ ZNS-Tuberkulose, ZNSMykose abklären.

- Liegt ein Pyozephalus vor? $\rightarrow$ eine bakterielle Meningitis abklären.

- Liegen multiple Hirnabszesse/Zerebritis, septische Herdenzephalitis vor? $\rightarrow$ an eine Endokarditis denken.

Dritter Schritt: Wenn die Gerinnungsparameter, vor allem die Thrombozytenzahl, massiv erniedrigt sind, sollte an virales hämorrhagisches Fieber mit ZNS-Beteiligung, z.B. Krim-Kongo-hämorrhagisches Fieber oder Hantavirus-Infektion, gedacht werden.

- Sind alle drei „Untersuchungsschritte“ unauffällig - Lumbalpunktion durchführen.
Vierter Schritt: Abklärung mittels Liquor cerebrospinalis inklusive mikrobiologischer, serologischer und molekularbiologischer gezielter Untersuchung des Serums und Liquors; Details sind der einschlägigen Standardliteratur zu entnehmen (z. B. [29, 32]). Aus infektionsepidemiologischen Gründen ist es sehr wichtig, durch präzise Anamnese einen Tollwutverdacht zu erhärten oder, soweit es geht, auszuschließen, da der Speichel und andere Körperflüssigkeiten (hoch) infektiös sein können. Rabies-Viren verursachen weltweit die höchste Zahl an tödlichen viralen Enzephalitiden.

- Liegt eine polymorphkernige Pleozytose vor, ist die Liquorglukose erniedrigt, das Laktat erhöht? $\rightarrow$ Gram-Färbung auf bakterielle Meningitis durchführen, vor allem bei Exposition im Meningitisgürtel Subsahara-Afrikas auf Meningokokken der Serogruppen A und W135 prüfen, beide sind im tetravalenten Meningokokkenimpfstoff enthalten.

- Liegt eine lymphozytäre Pleozytose vor, ist die Glukose \pm normal? $\rightarrow$ virale PCR („,polymerase chain reaction“), Tuschepräparat, Ziehl-Neelsen-Färbung, auf Mykobakterien untersuchen, Immunglobuline im Liquor untersuchen.

- Liegt eine eosinophile Pleozytose vor? $\rightarrow$ Larva migrans visceralis durch Angiostrongylus spp., Gnathostoma spp., Trichinella spp. abklären.

- Liquor normal $\rightarrow$ an zerebrale Malaria denken und mittels Blutausstrich abklären (typische Plasmodium falciparum [intraerythrozytäre] Ringformen im Blutausstrich - Abb. 1)

\section{Subakute Bewusstseinsstörung (quantitative und/oder qualitative) ohne Fieber}

Metabolische Erkrankungen (Diabetes mellitus, hepatische, urämische Enzephalopathie, etc.); endokrine Erkrankungen (Hyperthyreose, Hypothyreose, Hyperparathyreoidismus, etc.); Intoxikation; Malnutrition (Endstadium eines Kwashiorkor, Vitamindefizienzen - insbesondere Vitamin B1, Nikotinamid); granulomatöse, zystische, maligne intrakranielle Raumforderungen; Hydrozephalus; nichtkonvulsiver Status epilepticus.

- Merke

Beim bewusstseinsgestörten Patienten mit Fieber: erster Schritt: Fundoskopie - Malaria-Retinopathie? Eine frühest mögliche Diagnose und Therapieeinleitung mit Artesunat intravenös ist der wichtigste Prognosefaktor bei zerebraler Malaria.

\section{Akute Kopfschmerzen}

Migräne; spontane Subarachnoidalblutung; Meningitis; Sinus-/ innere Hirnvenenthrombose; hitzeassoziierte Kopfschmerzen; Hydrocephalus occlusus. Das Risiko einer Sinus-/inneren Hirnvenenthrombose wird - nach einer zur Exsikkose führenden Reisediarrhö - durch einen Langstreckenflug aggraviert.

\section{Nackensteifigkeit/Meningismus ohne Fieber}

Tetanus; spontane Subarachnoidalblutung; Dissektion der hirnzuführenden Gefäße: Gerade ältere/alte Menschen - mehr als 3\% der Fernreisenden sind älter als 70 Jahre - verlieren den Impf- 


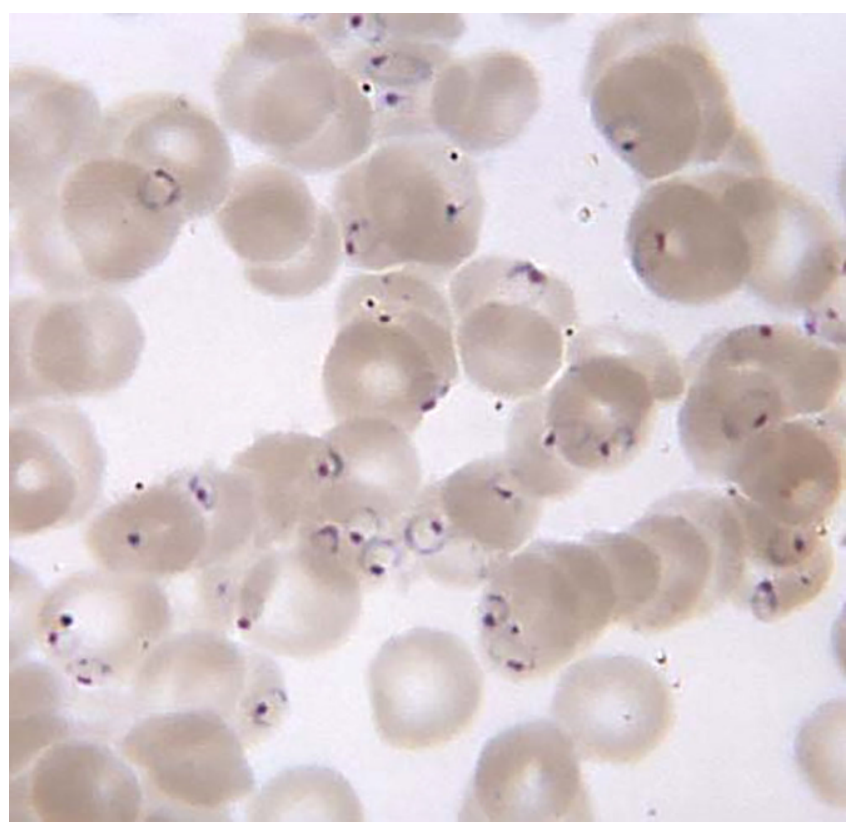

Abb. 1 A Blutausstrich bei zerebraler Malaria mit Multiorganversagen und Hyperparasitämie, ca. 50\% der Erythrozyten sind mit Trophozoiten („Ringformen") parasitiert

schutz, auch und besonders gegen Tetanustoxin. Clostridium tetani kommt weltweit vor.

- Merke

Tetanus nicht vergessen!

\section{Epileptische Anfälle mit Fieber}

Zerebrale Malaria; virale oder parasitäre Meningoenzephalitis; hitzeassoziierte Erkrankungen.

\section{Epileptische Anfälle ohne (mit wenig) Fieber}

Neurozystizerkose; Sinus-/innere Hirnvenenthrombose; granulomatöse/zystische/neoplastische/maligne Raumforderung; Residualepilepsie: Die Neurozystizerkose ist die häufigste Ursache einer sekundären Epilepsie, in Teilen Afrikas, Asiens und Lateinamerikas wird die Zahl der NeurozystizerkosepatientInnen auf bis zu 50 Mio. geschätzt. Eine rezente prospektive randomisierte, doppelblinde Studie konnte klar zeigen, dass mit der Kombinationstherapie Albendazol plus Praziquantel die Zahl der Zysten und vor allem die Zahl der epileptischen Anfälle hochsignifikant gesenkt werden kann.

\section{$\checkmark$ Merke}

Epilepsien und epileptische Anfälle sind häufig in vielen Herkunftsländern von MigrantInnen: Neurozystizerkose ist eine wichtige Differenzialdiagnose und ursächlich behandelbar (Kombinationstherapie mit Albendazol und Praziquantel).
Plötzlich auftretende Herdsymptomatik

Ischämischer, hämorrhagischer Schlaganfall; Todd-Parese; evtl. Hirnabszess.

\section{Syndrom der chronischen Meningitis oder Enzephalitis}

Bei Meningitis: subfebrile Temperaturen, Kopfschmerzen mit oder ohne Hirnnervenausfälle; bei Enzephalitis: üblicherweise plus diffuse oder fokale neurologische Funktionsstörung (z. B. Trypanosoma-bedingte Schlafkrankheit in Subsahara-Afrika).

ZNS-Tuberkulose; Kryptokokkose; Meningeosis neoplastica; Neurobrucellose; Afrikanische Trypanosomiasis (Infektion mit Trypanosoma brucei gambiense oder rhodesiense - westafrikanische bzw. ostafrikanische Schlafkrankheit); Larva migrans visceralis (Eosinophilie).

Die häufigste chronische Meningitis bei MigrantInnen und Flüchtlingen - mit und ohne HIV (humanes Immundefizienzvirus) -Infektion - ist die ZNS-Tuberkulose. Aus Südafrika, Russland sowie den Nachfolgestaaten der früheren UdSSR (Union der Sozialistischen Sowjetrepubliken) werden die höchsten Inzidenzen an MDR („,multidrug resistant ${ }^{\prime \prime}$ ) und XDR (,extended drug resistant ${ }^{\prime \prime}$ ) -Mykobakterien berichtet.

$>$ Merke

ZNS-Tuberkulose bei MigrantInnen: an MDR und XDR denken!

\section{Hirnnervenaffektionen}

Basale Meningitis (siehe chronische Meningitis); Hirnnervenneuritis.

Trauma/Zustand nach Trauma, Neurotoxine (z.B. Schlangenbiss), Botulismus, Wernicke-Enzephalopathie, Hirnstammaffektionen (entzündlich, ischämisch, hämorrhagisch, Raumforderung).

Extrapyramidale (primär neurodegenerative und sekundäre) Symptomatik

Idiopathisches Parkinson-Syndrom ist in den Tropen selten; symptomatisches Parkinson-Syndrom kann bedingt sein durch eine chronische Intoxikation z. B. im westlichen Indien mit Mangan oder auf der pazifischen Insel Guam durch BOAA ( $\beta$-Oxalylaminoalanin; "Guam disease“ [Parkinson-Syndrom plus Demenz plus amyotrophe Lateralsklerose]), wird gesehen nach arboviraler Enzephalitis: postenzephalitisches Parkinson-Syndrom (überwiegend nach durch Arboviren bedingter Enzephalitis, postinfektiös) und auch bei/nach durch Autoimmunantikörper mediierter Enzephalitis.

\section{Akute spinale Symptomatik}

Trauma, akute Querschnittsmyelitis post-, parainfektiös, postvakzinal; Poliomyelitis und andere enterovirale (Polio-)Myelitiden, z. B. Enterovirus 68 (69, 70, 71) -Infektion; eosinophile Myelitis (Gnathostomiasis); Spondylitis verschiedener Ursachen mit vaskulär ischämischer Myelonschädigung bzw. Fortschreiten des entzünd- 
lichen Prozesses in den Spinalkanal. Die Problematik der akuten Myelitis/Radikulomyelitis bei einer Reiserückkehrerin wird in der Kasuistik diskutiert.

\section{> Merke}

Liquoreosinophilie: Larva migrans visceralis bei Meningitis, Hirnnervenneuritis und vor allem Radikulomyelitis; gut behandelbar, wenn rechtzeitig diagnostiziert (Albendazol).

\section{Subakute, chronische Querschittssymptomatik}

Spinale Raumforderungen (extra- und intramedullär); tropische Myeloneuropathien (nutritiv bedingt: Konzo, Lathyrismus); tropische spastische Paraparese (HTLV I [humanes T-lymphotropes Virus] -assoziierte Myelopathie - HAM); Fluorose/Vertebrostenose; funikuläre Myelose (Vitamin-B-12-/Folsäuremangel); amyotrophe Lateralsklerose.

\section{Symptomatische Myopathie/Myositis}

Fokale Myositis (Larva migrans Infektionen, Trichinose); Neurozystizerkose; Rhabdomyolyse nach Schlangenbissen.

\section{Polyneuropathie}

Metabolisch/toxisch bedingt (Diabetes mellitus, alkoholassoziiert); inflammatorische Polyradikuloneuropathie (Guillain-BarréSyndrom), in Westafrika häufig HIV-assoziiert; Hypovitaminosen (insbesondere Vitamin B-Gruppe); infektiöse Ursachen (Borrelien, Enteroviren, Retroviren, Lepra, Rabies); tropische/ataktische Neuropathie (Vitamin-B-Gruppe); hereditär bedingte periphere Neuropathien.

\section{Radikuläre Symptomatik, periphere Nervenläsion}

Infektiöse Radikulitis (Borrelien spp., Herpes-Zoster-Viren); Radikulopathie bei Gnathostoma-spinigerum-Infestation; Plexusneuritis (z.B. Schistosomiasis); Lepra; raumfordernde, intra- und extradurale Prozesse (Granulome, Diskusprolaps etc.).

\section{Fazit für die Praxis}

- Fernreisende, MigrantInnen und Flüchtlinge zeigen unterschiedliche „importierte“ Krankheitsmuster.

- Akute Infektionen des Zentralnervensystems (ZNS), wie virale Enzephalitis und zerebrale Malaria, werden bei Fernreiserückkehrern und subakute/chronische Erkrankungen bei MigrantInnen häufiger gesehen.

- Bei lebensbedrohlicher akuter ZNS-Infektion sollte als erster Schritt eine Fundoskopie die sofortige weiterführende Diagnostik und Therapie (Plasmodium-falciparum-Malaria - i.v. Artesunat) erlauben.

- Bei MigrantInnen aus weiten Teilen Afrikas und Lateinamerikas sowie Süd- und Südostasiens ist die Neurozystizerkose die häufigste Epilepsieursache. Mittels Bildgebung kann sie schnell diagnostiziert werden; sie ist gut mit einer Kombination von Albendazol und Praziquantel therapierbar.
- Bei älteren Menschen ist im Einzelfall auch heute noch Tetanus eine wichtige Differenzialdiagnose.

Korrespondenzadresse

Univ. Prof. Dr. Erich Schmutzhard

Universitätsklinik für Neurologie, Medizinische Universität Innsbruck Anichstraße 35, 6020 Innsbruck, Österreich

erich.schmutzhard@i-med.ac.at

Funding. Open access funding provided by University of Innsbruck and Medical University of Innsbruck.

\section{Einhaltung ethischer Richtlinien}

Interessenkonflikt. Gemäß den Richtlinien des Springer Medizin Verlags werden Autoren und Wissenschaftliche Leitung im Rahmen der Manuskripterstellung und Manuskriptfreigabe aufgefordert, eine vollständige Erklärung zu ihren finanziellen und nichtfinanziellen Interessen abzugeben.

Autoren. E. Schmutzhard: A. Finanzielle Interessen: Vortragshonorar, Reisekostenerstattung:ZOLL Medical. - Bezahlter Berater/interner Schulungsreferent/ Gehaltsempfänger o. Ä.: Pfizer, Bexsero. - B. Nichtfinanzielle Interessen: Angestellter Neuro-Intensivist, Medizinische Universität Innsbruck, Österreich, seit Oktober 2017 im Ruhestand | Lehrbeauftragter am Bernhard Nicht Institut für Tropenmedizin, Hamburg, Deutschland|Mitgliedschaften folgender Komitees beim Österreichischen Bundesministerium für Arbeit, Soziales, Gesundheit und | Konsumentenschutz (BMASGK): Polio-Eradikation, Masern \& Röteln, Rabies/Tollwut|Mitgliedschaften: OGN, DGN, DGNI, OGIT, OGTPM, DTG. B. Pfausler: A. Finanzielle Interessen: Teilnahmegebühren und Reiskostenerstattung für ECCMID-Besuch: Fa. Pfizer| Kongressunterstützung (Kongressgebühr, Reise, Hotel): Fa. Pfizer, Fa. EVER Neuro Pharma|Honorar für Vortrage: Zoll, Amomed. - B. Nichtfinanzielle Interessen: Angestellte Neuro-Intensivistin, Medizinische Universität Innsbruck, Österreich | Mitgliedschaften: ÖGN, ÖGIT, EAN.

Wissenschaftliche Leitung. Die vollständige Erklärung zum Interessenkonflikt der Wissenschaftlichen Leitung finden Sie am Kurs der zertifizierten Fortbildung auf www. springermedizin.de/cme.

Der Verlag erklärt, dass für die Publikation dieser CME-Fortbildung keine Sponsorengelder an den Verlag fließen.

Für diesen Beitrag wurden von den Autoren keine Studien an Menschen oder Tieren durchgeführt. Für die aufgeführten Studien gelten die jeweils dort angegebenen ethischen Richtlinien.

Open Access. Dieser Artikel wird unter der Creative Commons Namensnennung 4.0 International Lizenz veröffentlicht, welche die Nutzung, Vervielfältigung, Bearbeitung, Verbreitung und Wiedergabe in jeglichem Medium und Format erlaubt, sofern Sie den/die ursprünglichen Autor(en) und die Quelle ordnungsgemäßnennen, einen Link zur Creative Commons Lizenz beifügen und angeben, ob Änderungen vorgenommen wurden.

Die in diesem Artikel enthaltenen Bilder und sonstiges Drittmaterial unterliegen ebenfalls der genannten Creative Commons Lizenz, sofern sich aus der Abbildungslegende nichts anderes ergibt. Sofern das betreffende Material nicht unter der genannten Creative Commons Lizenz steht und die betreffende Handlung nicht nach gesetzlichen Vorschriften erlaubt ist, ist für die oben aufgeführten Weiterverwendungen des Materials die Einwilligung des jeweiligen Rechteinhabers einzuholen.

Weitere Details zur Lizenz entnehmen Sie bitte der Lizenzinformation auf http:// creativecommons.org/licenses/by/4.0/deed.de. 


\section{Literatur}

\section{Verwendete Literatur}

1. World Tourism Organization (UNWTO) (2019) Weltweite internationale „tourist arrivals" im Jahre 2018. https://www.untwo.org. Zugegriffen: 28. September 2019

2. World Tourist Organization (UNWTO) (2019) Entwicklung der weltweiten Reisen („tourist-arrivals"), 1950 bis 2018, mit Vorausschätzung für die nächsten 12 Jahre. https://www.untwo.org. Zugegriffen:28. September 2019

3. United Nations Department of Economic and Social Affairs (UN DESA) (2019) https://www.un.org/development/desa/. Accessed 29. September 2019

4. Brinckmann MP, van Noort BM, Leithner C, Ploner CJ (2018) Neurological emergencies in refugees. Front Neurol 9:1088. https://doi.org/10.3389/fneur. 2018.01088 (eCollection 2018)

5. Senadim S, Uygun E, Erdogan M, Koksal A, Soysal A, Atakli D (2018) Profile of Syrian asylum-seekers from neurological clinic in a tertiary center. Eur Neurol 80(5-6):249-253

6. Kluge U, Rapp MA, Mehran N, Jumaa J, Aichberger MC (2019) Armut, Migration und psychische Gesundheit. Nervenarzt. https://doi.org/10.1007/s00115-019-007902 (published online 26 Sept 2019)

7. Badri A, Crutzen R, Van den Borne HW (2012) Exposures to war-related traumatic events and post-traumatic stress disorder symptoms among displaced Darfuri female university students: an exploratory study. Bmc Public Health 12:603. https://doi.org/10.1186/1471-2458-12-603

8. Liu D, Fu L, Jing Z, Chen C (2016) Post-traumatic stress disorder and it's predictors among Tibetan adolescents 3 years after the high-altitude earthquake in China. Arch Psychiatr Nurs 30:593-599

9. Fölsch UR, Hasenfuss G, Spies HF, Wesiack W, Faulbaum F (2016) Flucht und Migration: Eine Herausforderung für die Medizin in Deutschland. Internist 57:822-830

10. Ekmekci PE (2017) Syrian refugees, health and migration legislation in Turkey. JImmigr Minor Health 6:1434-1441

11. Ding H, Hargraves L (2009) Stress-associated poor health among adult immigrants with a language barrier in the United States. J Immigr Minor Health 11:446-452

12. Khan MS, Osei-Kofi A, Omar A et al (2016) Pathogens, prejudice, and politics: the role of the global health community in the European refugee crisis. Lancet Infect Dis 16:e173-e177

13. Burton A (2019) Meeting the neurological needs of refugees and other forcibly displaced people. Lancet Neurol 18(6):524-525. https://doi.org/10.1016/S14744422(19)30164-4

14. Müller M, Khamis D, Srivastava D, Exadaktylos AK, Pfortmueller CA (2018) Understanding refugees' health. Semin Neurol 38(2):152-162. https://doi.org/10. 1055/s-0038-1649337

15. Pfausler B, Schmutzhard E (2017) Flüchtlinge in der Neurologie: Importierte Erkrankungen des Nervensystems erkennen und behandeln. Psychiatr Neurol $1: 18-22$

16. Robert-Koch-Institut (2016) Dem Robert-Koch-Institut übermittelte meldepflichtige Infektionskrankheiten bei Asylsuchenden in Deutschland; August 2016 (31.-35. Kalenderwoche). https://www.rki.de/.../Asylsuchende/.../ meldepflichtige_Infektionskrankheiten.Zugegriffen:21.Sept. 2016

17. Beermann S, Rexroth U, Kirchner M, Kühne A, Vygen S, Gilsdorf A (2015) Asylsuchende und Gesundheit: Epidemiologisch relevante Infektionskrankheiten. Dtsch Arztebl 112:1717-1721

18. Stich A (2016) Häufige Infektionskrankheiten bei Migranten. Internist 57:409-415

19. Monge-Maillo B, López-Vélez R, Norman FF, Ferrere-González F, Martínez-Pérez Á, Pérez-Molina JA (2015) Screening of imported infectious diseases among asymptomatic sub-Saharan African and Latin American immigrants: a public health challenge. Am JTrop Med Hyg 92:848-856

20. Kuna A, Gajewski M, Biernat B (2019) Selected arboviral diseases imported to Poland-current state of knowledge and perspectives for research. Ann Agric Environ Med 26(3):385-391

21. Norman FF, Monge-Maillo B, Martínez-Pérez Á, Perez-Molina JA, López-Vélez R (2015) Parasitic infections in travelers and immigrants: part I protozoa. Future Microbiol 10(1):69-86. https://doi.org/10.2217/fmb.14.105 (Review)

22. Norman FF, Monge-Maillo B, Martínez-Pérez Á, Perez-Molina JA, López-Vélez $R$ (2015) Parasitic infections in travelers and immigrants: part II helminths and ectoparasites. Future Microbiol 10(1):87-99. https://doi.org/10.2217/fmb.14.106 (Review)

23. Pérez-Arellano JL, Górgolas-Hernández-Mora M, Salvador F et al (2018) Executive summary of imported infectious diseases after returning from foreign travel: consensus document of the Spanish Society for Infectious Diseases and Clinical Microbiology (SEIMC). Enferm Infecc Microbiol Clin 36(3):187-193. https://doi. org/10.1016/j.eimc.2017.02.009
24. Monge-Maillo B, López-Vélez R, Norman FF, Ferrere-González F, Martínez-Pérez Á, Pérez-Molina JA (2015) Screening of imported infectious diseases among asymptomatic sub-Saharan African and Latin American immigrants: a public health challenge. Am J Trop Med Hyg 92(4):848-856

25. Sauerbier A, Macerollo A, Györfi $O$ et al (2016) Insufficient global health education in European neurological post-graduate training: a European Association of Young Neurologists and Trainees survey. Eur J Neurol 23(11):1680-1683

26. PfauslerB, VollertH,BöschS, SchmutzhardE(1996)Cerebralvenousthrombosis-a new diagnosis in travel medicine? J Travel Med 3(3):165-167

27. Thakur K, Zunt J (2011) Neurologic parasitic infections in immigrants and travelers. Semin Neurol 31:231-244

28. O'Brien DP, Leder K, Matchett E, Brown GV, Torresi J (2006) Illness in returned travelers and immigrants/refugees: the 6-year experience of two Australian infectious diseases units. JTravel Med 13:145-152

29. Schmutzhard E, Löscher T (2015) Neurologie in den Tropen. In: Löscher T, Burchard GD (Hrsg) Tropenmedizin in Klinik und Praxis, 4. Aufl. Thieme, Stuttgart, S228-235

30. Kühne A, Gilsdorf A (2016) Ausbrüche von Infektionskrankheiten in Gemeinschaftsunterkünften für Asylsuchende 2004-2014 in Deutschland. Bundesgesundheitsblatt Gesundheitsforschung Gesundheitsschutz 59:570-577

31. Zur B (2016) Zunahme genetisch determinierter Anämien durch Migration in Deutschland. Internist 2016(57):444-451

32. Suerbaum S, Burchard GD, Kaufmann SHE, Schulz TF (Hrsg) (2016) Medizinische Mikrobiologie und Infektiologie(Springer-Lehrbuch), 8. Aufl.Springer, Heidelberg, Berlin (Kapitel 113und 126)

33. Burton M, Nyong'o O, Burton K et al (2004) Retinopathy in Gambian children admitted to hospital with malaria. Trop Doct 34:214-218

\section{Weiterführende Literatur}

34. Bundesamt für Migration und Flüchtlinge (BAMF) (2019) https://www.bamf.de. Accessed 29. September 2019 
? Ein 18-jähriger Flüchtling aus Afghanistan mit $39,6^{\circ} \mathrm{C}$ Fieber, generalisiertem Anfall, mäßiger Nackensteifigkeit und Petechien bei ausgeprägter Thrombopenie. Im kranialen Computertomogramm (cCT; siehe unten): frontale sulkale Subarachnoidalblutung: kein Trauma. Auf der Flüchtlingsroute häufiger Kontakt mit Ratten, Rattenurin. Welche Diagnose ist am wahrscheinlichsten?

O Zerebrale Malaria durch Plasmodium vivax

○ Hämorrhagisches Fieber mit ZNS (Zentralnervensystem) -Affektion durch Hantaviren

O Posteriores reversibles Enzephalopathiesyndrom

O Japanische Enzephalitis

O Meningokokkenmeningitis und -sepsis

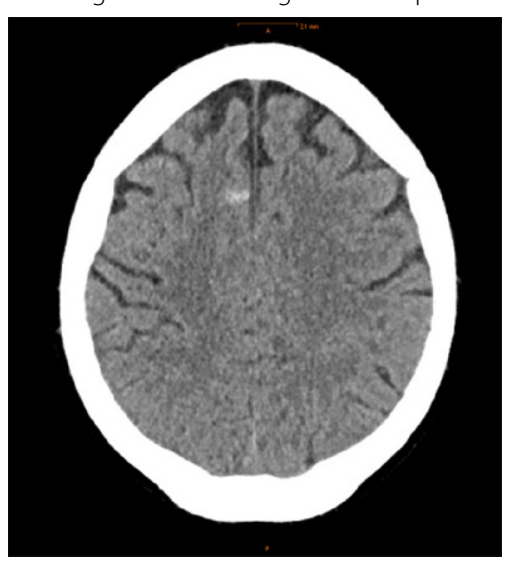

? Was ist die häufigste Ursache einer eosinophilen Radikulomyelitis in Südostasien?

O Coccidioides immitis

O Cysticercus cellulosae

○ Dengue-Viren

O Gnathostoma spinigerum

○ Bartonella henselae

Ein 62-jähriger deutscher Krankenhaustechniker (in Uganda) - auf Heimaturlaub - erkrankt mit Kopfschmerzen, Gliederschmerzen, Durchfall und sehr hohem irregulärem Fieber. Nach einem generalisierten epileptischen Anfall wird er komatös, Glasgow Coma Scale (GCS) 6, in die Klinik eingeliefert. Die notfallmäßig durchgeführte Fundoskopie zeigt multiple kleine Retinablutungen, vermischt mit ischämischen Arealen. Was ist Ihre Diagnose?

○ Zerebrale Malaria durch Plasmodium falciparum

Ebola-Virus-Infektion

O Vaskulitis autoimmuner Genese

West-Nil-Virus-Infektion

○ Babesiose
Eine 25-jährige Migrantin (vor 4 Jahren aus Tschetschenien immigriert) klagt seit 6 Wochen über Kopfschmerzen, subfebrile Temperaturen, intermittierendes Erbrechen und nun seit 2 Wochen über Doppelbilder. Der Neurostatus zeigt einen milden Meningismus, eine Hyperreflexie sowie eine N.-abducens-Parese rechts und eine milde bilaterale N.-facialis-Parese. Worauf müssen Sie bei der klinischen Verdachtsdiagnose einer tuberkulösen Meningitis bei diesem Fall besonders achten?

O Konkomittierende HIV-Infektion

O Begleithydrozephalus

O Zugrunde liegende maligne Erkrankung

○ Resistenzsituation von M. tuberculosis in ihrem Herkunftsland

○ Andere opportunistische Infektion

Woran denken Sie bei Kiefersperre und Nackensteifigkeit beim alten Menschen?

O Kiefergelenksarthrose

O Hirnnervenneuritis

O Arteritis temporalis

O Pneumokokkenmeningitis

O Tetanus

\section{Informationen zur zertifizierten Fortbildung}

Diese Fortbildung wurde von der Ärztekammer Nordrhein für das „Fortbildungszertifikat der Ärztekammer" gemäß $§ 5$ ihrer Fortbildungsordnung mit 3 Punkten (Kategorie D) anerkannt und ist damit auch für andere Ärztekammern anerkennungsfähig.
Anerkennung in Österreich: Für das Diplom-Fortbildungs-Programm (DFP) werden die von deutschen Landesärztekammern anerkannten Fortbildungspunkte aufgrund der Gleichwertigkeit im gleichen Umfang als DFP-Punkte anerkannt (§ 14, Abschnitt 1, Verordnung über ärztliche Fortbildung, Österreichische Ärztekammer (ÖÄK) 2013).
Hinweise zur Teilnahme:

- Die Teilnahme an dem zertifizierten Kurs ist nur online auf www.springermedizin.de/cme möglich.

- Der Teilnahmezeitraum beträgt 12 Monate. Den Teilnahmeschluss finden Sie online beim Kurs.

- Die Fragen und ihre zugehörigen Antwortmöglichkeiten werden online in zufälliger Reihenfolge zusammengestellt.
- Pro Frage ist jeweils nur eine Antwort zutreffend.

- Für eine erfolgreiche Teilnahme müssen $70 \%$ der Fragen richtig beantwortet werden.

- Teilnehmen können Abonnenten dieser Fachzeitschrift und e.Med- und e.Dent-Abonnenten. 
(3) Ein 24-jähriger indischer Student mit erstmals aufgetretenen fokal motorischen, sekundär generalisierten epileptischen Anfällen zeigt im kranialen Computertomogramm (cCT) 3 zystische Läsionen mit intrazystischer Hyperdensität. Was schließt Ihre Differenzialdiagnose auf jeden Fall mit ein?

Tsutsugamushi-Fieber

○ Sichelzellanämie

○ Neurozystizerkose

○ Zika-Virus-Infektion

○ Zustand nach Schädel-Hirn-Trauma

? Welche virale Enzephalitis verursacht mit knapp 60.000 die höchste Zahl an Todesfällen weltweit?

○ Gelbfieber-Viren

○ Dengue-Fieber-Viren

O Lyssa-Viren

O Japanische Enzephalitisviren

○ FSME (Frühsommer-Meningoenzephalitis) -Viren

? Welches sind die häufigsten Erreger einer chronischen Enzephalitis in Subsahara-Afrika?

O Masern-Viren

O Marburg-Viren

O Meningokokken Serotyp W135

O Kryptokokken

- Trypanosomabrucei

? Welche chemische Substanz verursacht in Teilen Indiens überdurchschnittlich häufig ein symptomatisches Parkinson-Syndrom?

O Asbest

O Quecksilber

○ $\beta$-Oxalylaminoalanin (BOAA)

O Mangan

O Arsen

? Welcher Meningokokken Serotyp ist nicht im für Subsahara-Afrika empfohlenen tetravalenten Impfstoff enthalten

O Serotyp A

O Serotyp $X$

O Serotyp $Y$

O Serotyp C

O Serotyp W 135 